\title{
Long-term survival and quality of life after intensive care for patients 80 years of age or older
}

Finn H Andersen ${ }^{1,2^{*}}$, Hans Flaatten ${ }^{3,4}$, Pål Klepstad ${ }^{5,2}$, Ulla Romild ${ }^{6,7}$ and Reidar Kvåle ${ }^{3}$

\begin{abstract}
Background: Comparison of survival and quality of life in a mixed ICU population of patients 80 years of age or older with a matched segment of the general population.

Methods: We retrospectively analyzed survival of ICU patients $\geq 80$ years admitted to the Haukeland University Hospital in 2000-2012. We prospectively used the EuroQol-5D to compare the health-related quality of life (HRQOL) between survivors at follow-up and an age- and gender-matched general population. Follow-up was 1-13.8 years.

Results: The included 395 patients (mean age 83.8 years, $61.0 \%$ males) showed an overall survival of 75.9 (ICU), 59.5 (hospital), and $42.0 \% 1$ year after the ICU. High ICU mortality was predicted by age, mechanical ventilator support, SAPS II, maximum SOFA, and multitrauma with head injury. High hospital mortality was predicted by an unplanned surgical admission. One-year mortality was predicted by respiratory failure and isolated head injury. We found no differences in HRQOL at follow-up between survivors $(n=58)$ and control subjects $(n=179)$ or between admission categories. Of the ICU non-survivors, $63.2 \%$ died within 2 days after ICU admission $(n=60)$, and $68.3 \%$ of these had life-sustaining treatment (LST) limitations. LST limitations were applied for $71.3 \%(n=114)$ of the hospital non-survivors (ICU $70.5 \%(n=67)$; post-ICU $72.3 \%(n=47))$.

Conclusions: Overall 1-year survival was $42.0 \%$. Survival rates beyond that were comparable to those of the general octogenarian population. Among survivors at follow-up, HRQOL was comparable to that of the age- and sex-matched general population. Patients admitted for planned surgery had better short- and long-term survival rates than those admitted for medical reasons or unplanned surgery for 3 years after ICU admittance. The majority of the ICU non-survivors died within 2 days, and most of these had LST limitation decisions.
\end{abstract}

Keywords: Intensive care unit; Elderly; Octogenarians; Survival; Mortality; HRQOL; Long-term outcome

\section{Background}

In many countries, aged populations may increase by $40-50 \%$ in the coming decades [1-3]. A similar increase is expected in the proportion of older patients admitted to intensive care units (ICU). Patients 80 years of age or older currently constitute between 8.9 and $13.8 \%$ of large national ICU registries [4-7]. Australia and New Zealand showed $5.6 \%$ annual increases in the numbers of

\footnotetext{
* Correspondence: finn.andersen@ntnu.no

'Department of Anesthesia and Intensive Care, Møre and Romsdal Health Trust, Ålesund Hospital, 6026 Ålesund, Norway

${ }^{2}$ Department of Circulation and Medical Imaging, Faculty of Medicine,

Norwegian University of Science and Technology, Pb. 8905, 7491

Trondheim, Norway

Full list of author information is available at the end of the article
}

octogenarians that entered the ICU [4]; in Denmark, an $18 \%$ increase was observed from 2005 to 2011 [5].

Few recent studies have focused on long-term healthrelated quality of life (HRQOL) in aged ICU survivors, and even fewer have compared octogenarian ICU patients to an older segment of the general population. These studies were mainly performed in medical ICUs and included small sample sizes, due to high short-term mortality $[8,9]$. One- and 2-year mortalities in octogenarians are reported to be as high as 72.0 and $79 \%$ [9], respectively. Thus, it is important to identify factors among the older population that predict benefit from ICU treatment, establish prognostic factors for longterm survival, and elucidate the HRQOL.

\section{实}


This study aimed to:

1. Compare survival and HRQOL between older patients and age-matched control groups from the general population;

2. Identify predictors for short- and long-term mortality among older ICU patients; and

3. Compare survival and HRQOL scores between the different SAPS II admission categories: admissions for planned surgery, unplanned surgery, and medical reasons.

\section{Methods}

Haukeland University Hospital is a tertiary university hospital in Bergen, Norway, which serves approximately one million inhabitants. The general ICU has ten beds (burn, cardiac surgery, coronary, and neonatal units are separate units, and are not included in this study). The annual number of admissions is about 500 , and $7-8 \%$ of patients are 80 years of age or older. There were no large changes in practice or organization of the ICU during the study period besides general development in medicine and intensive care.

\section{Study design}

The first part of this study was a retrospective analysis of patients $\geq 80$ years old, which were admitted to this general ICU between the 1st of January 2000 and the 31st of December 2012. These data were extracted from the dedicated ICU database with daily, prospectively collected data. Re-admissions, non-Norwegian patients, and admissions with errors in patient ID were excluded. For all included patients, we assessed the following:

1. Age and gender;

2. Length of stay (LOS);

3. Ventilator support, invasive (mechanical) and noninvasive ventilator support;

4. Severity score (simplified acute physiology score II (SAPS II) [10]) and organ dysfunction (sequential organ failure assessment score (SOFA) [11]): we defined severe organ dysfunctions as a SOFA score of 3 or 4; among daily SOFA scores, only the maximum was included in the analysis;

5. Comorbidity: we separated comorbidity in four categories (none, mild, moderate, and severe) based on the Charlson comorbidity index (CCI) [12];

6. Diagnostic groups: ICU admissions were allocated into one of thirteen different categories;

7. Short- and long-term survival (long-term defined as 1 year and longer): the standardized mortality ratio (SMR) was defined as the observed hospital mortality divided by the SAPS II estimated mortality; the SMR was analyzed for all patients and for each of the SAPS II admission categories;
8. Survival at follow-up; and

9. SAPS II admission categories, planned surgery, unplanned surgery, and medical reasons.

Survival was compared with a segment of the general population that was 80 years of age or older during 2000 2013, based on life tables from Statistics Norway.

The second part of the study included a prospective analysis of HRQOL. Patients alive at follow-up (16th of January 2014) were compared with a control group of 375 individuals matched for age, sex, and residence, which were randomly drawn from the National Registry. The HRQOL was assessed with EuroQol-5D (EQ-5D-3L) [13], a questionnaire sent by mail to ICU survivors and the control group at follow-up. EQ-5D has five dimensions, each with three response options. It also included a visual analog scale (EQ-VAS; Table 4). A reminder was sent to the non-responders after 1 month. ICU survivors were also contacted by phone. Informed consent was given by persons who answered the questionnaire.

We compared hospital survivors with hospital nonsurvivors and also compared the SAPS II admission categories. Information about end-of-life decision-making was retrospectively found for hospital non-survivors by searching through the individual patient files of their current hospital stay since such information was not entered in the ICU database. We only included statements which clearly used the terms withholding or withdrawal of ICU treatment.

The study was approved by the Regional Committee of Medical and Health Research Ethics in Central Norway (REC Central, 2013/1113).

\section{Statistics}

The length of stay (LOS) and ventilator time are expressed in terms of medians and quartiles. Significance was tested with the Mann-Whitney U test/Kruskal-Wallis test. Other continuous variables are expressed as the mean with standard deviation (SD) and compared with the $t$ test/analysis of variance (ANOVA). Qualitative and dichotomous data are reported as the percent of $n$, and they were compared with Pearson's chi-square test/Fisher's exact test or with the Mann-Whitney U test. Three separate Cox proportional hazard regression analyses were used to determine independent predictors of ICU mortality, hospital mortality, and 1-year mortality. The time factor was defined as the number of days from ICU admission, ICU discharge, and hospital discharge, respectively. All variables with a $p$ value of $<0.2$ in a primary univariate analysis were included in the multivariate model, except for admission categories; admission categories were included even when the $p$ value was $>0.2$ in the univariate analysis. ICU mortality was analyzed separately. Only ICU survivors were included in the analysis of hospital mortality. Only hospital survivors were 
included in the 1-year mortality analysis. The remaining variables were then tested separately in the models, and included if they were significant. Adjusted hazard ratios (HR) were calculated with $95 \%$ confidence intervals (95\% CI). Kaplan-Meier curves were constructed from the three SAPS II admission categories. Another Kaplan-Meier curve was constructed to compare all patients to the general octogenarian population in Norway. An adjusted mortality rate was calculated by dividing the observed mortality rate by the expected mortality rate from an age- and gender-matched population. The adjusted mortality rate was calculated between 1 and 8 years after ICU admission. Patients who were alive at follow-up were censored. All statistical analyses were performed with SPSS 21.0 (SPSS Inc., Chicago, IL, USA). P values $<0.05$ were considered statistically significant.

\section{Results}

From 2000 to 2012, 402 patients $\geq 80$ years were admitted to our ICU, with a total of 419 ICU stays. Re-admissions (during the same hospital stay $(n=10)$ and during another later hospital stay $(n=7))$, non-Norwegian patients $(n=$ $4)$, and admissions with errors in patient ID $(n=3)$ were omitted from the analysis. Thus, 395 patients were included in the current study (Fig. 1).

\section{Patient characteristics}

\section{Age and gender}

At ICU admittance, the mean age was 83.8 years (range 80-101; median 83.1) and $61.0 \%$ were males (Table 1 ). Males had longer median ICU-LOS (2.1 vs. 1.5 days, $p=$ $0.006)$, a higher mean maximum SOFA score (8.3 vs. 7.0 ,

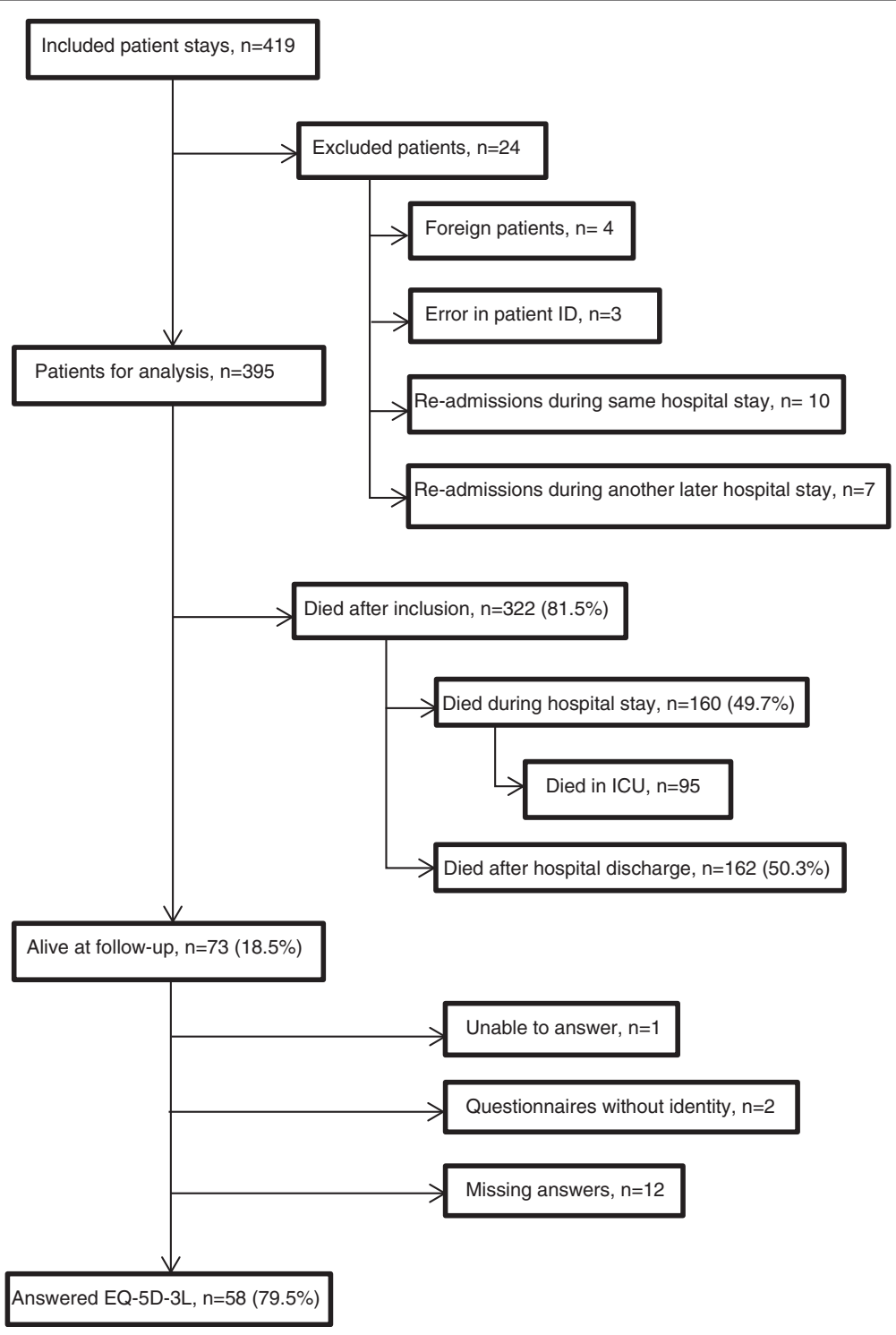

Fig. 1 Data collection process 
Table 1 Differences in characteristics between hospital survivors and hospital non-survivors

\begin{tabular}{|c|c|c|c|c|}
\hline & Total $(n=395)$ & Hospital survivors $(n=235)$ & Hospital non-survivors $(n=160)$ & $p$ value \\
\hline Age, mean $\pm S D$ & $83.8 \pm 2.9$ & $83.5 \pm 2.9$ & $84.1 \pm 2.8$ & $0.049^{a}$ \\
\hline Male, \% & 61.0 & 60.9 & 61.3 & $0.511^{b}$ \\
\hline \multicolumn{5}{|l|}{ Length of stay (LOS), median (IQR) } \\
\hline ICU-LOS & $1.8(0.9-3.9)$ & $1.9(1.0-4.3)$ & $1.7(0.8-3.2)$ & $0.097^{c}$ \\
\hline Hospital LOS & $11.3(4.0-19.3)$ & $14.2(7.6-25.1)$ & $5.5(1.9-12.8)$ & $<0.001^{\mathrm{c}}$ \\
\hline \multicolumn{5}{|l|}{ Ventilator support } \\
\hline Mechanical ventilator support, \% (n) & $61.3(242)$ & $51.9(122)$ & $75.0(120)$ & $<0.001 \mathrm{~b}$ \\
\hline Mechanical ventilator support time, median (IQR) & $1.2(0.5-3.3)$ & $1.3(0.5-3.8)$ & $1.0(0.4-3.0)$ & $0.235^{c}$ \\
\hline Non-invasive ventilator support, \% (n) & $33.2(131)$ & $35.8(84)$ & $29.6(47)$ & $0.344^{b}$ \\
\hline Non-invasive ventilator support time, median (IQR) & $1.5(0.5-2.8)$ & $1.6(0.5-3.2)$ & $1.3(0.4-2.5)$ & $0.164^{c}$ \\
\hline \multicolumn{5}{|l|}{ Severity score, mean \pm SD } \\
\hline SAPS $\|$ & $44.3 \pm 15.0(n=390)$ & $40.6 \pm 12.9(n=230)$ & $49.5 \pm 16.3(n=160)$ & $<0.001^{\mathrm{a}}$ \\
\hline Max. SOFA & $7.8 \pm 3.8(n=389)$ & $6.7 \pm 3.3(n=229)$ & $9.5 \pm 3.8(n=160)$ & $<0.001^{a}$ \\
\hline \multicolumn{5}{|l|}{ Comorbidity } \\
\hline Charlson comorbidity index, mean \pm SD & $2.6 \pm 1.9(n=390)$ & $2.7 \pm 1.8(n=234)$ & $2.5 \pm 1.9(n=156)$ & $0.389^{a}$ \\
\hline Charlson comorbidity index (CCl) categories, \% (n) & & & & $0.602^{b}$ \\
\hline None (CCl 0) & $12.3(48)$ & $11.1(26)$ & $14.1(22)$ & \\
\hline Mild (CCl 1-2) & $40.8(159)$ & $39.3(92)$ & $42.9(67)$ & \\
\hline Moderate (CCl 3-4) & $32.3(126)$ & $34.2(80)$ & $29.5(46)$ & \\
\hline Severe $(\mathrm{CCl} \geq 5)$ & $14.6(57)$ & $15.4(36)$ & $13.5(21)$ & \\
\hline \multicolumn{5}{|l|}{ Severe organ dysfunction, $\%(n)$} \\
\hline Respiration & $66.3(262)$ & $62.6(147)$ & $71.9(115)$ & $0.034^{b}$ \\
\hline Circulation & $47.1(186)$ & $38.7(91)$ & $59.4(95)$ & $<0.001^{\mathrm{b}}$ \\
\hline Renal & $28.1(111)$ & $20.9(49)$ & $38.8(62)$ & $<0.001^{\mathrm{b}}$ \\
\hline CNS & $26.1(103)$ & $18.7(44)$ & $36.9(59)$ & $<0.001^{\mathrm{b}}$ \\
\hline Coagulation & $9.9(39)$ & $9.8(23)$ & $10.0(16)$ & $0.537^{b}$ \\
\hline Liver & $1.3(5)$ & $0.9(2)$ & $1.9(3)$ & $0.399^{d}$ \\
\hline Admission categories, \% ( $n)$ & & & & $<0.001^{\mathrm{b}}$ \\
\hline Planned surgery & $12.7(50)$ & $17.9(42)$ & $5.0(8)$ & \\
\hline Unplanned surgery & $53.9(213)$ & $49.8(117)$ & $60.0(96)$ & \\
\hline Medical reasons & $33.4(132)$ & $32.3(76)$ & $35.0(56)$ & \\
\hline \multicolumn{5}{|l|}{ Diagnostic groups, \% (n) } \\
\hline Respiratory failure & $28.1(111)$ & $31.1(73)$ & $23.8(38)$ & $0.112^{b}$ \\
\hline Circulatory failure & $8.1(32)$ & $7.7(18)$ & $8.8(14)$ & $0.697^{b}$ \\
\hline Combined respiratory and circulatory failure & $10.4(41)$ & $8.9(21)$ & $12.5(20)$ & $0.254^{b}$ \\
\hline Neurologic failure & $10.1(40)$ & $9.4(22)$ & $11.3(18)$ & $0.541^{b}$ \\
\hline Isolated head injury & $2.5(10)$ & $1.7(4)$ & $3.8(6)$ & $0.328^{d}$ \\
\hline Sepsis & $8.9(35)$ & $7.2(17)$ & $11.3(18)$ & $0.168^{b}$ \\
\hline Gastroenterological failure & $4.8(19)$ & $4.7(11)$ & $5.0(8)$ & $0.884^{b}$ \\
\hline Multiple organ failure & $5.6(22)$ & $3.0(7)$ & $9.4(15)$ & $0.007^{b}$ \\
\hline Multitrauma without head injury & $3.8(15)$ & $5.1(12)$ & $1.9(3)$ & $0.099^{b}$ \\
\hline Multitrauma with head injury & $2.3(9)$ & $1.7(4)$ & $3.1(5)$ & $0.495^{\mathrm{d}}$ \\
\hline
\end{tabular}


Table 1 Differences in characteristics between hospital survivors and hospital non-survivors (Continued)

\begin{tabular}{lllll}
\hline Planned surgery & $3.3(13)$ & $5.5(13)$ & $0.0(0)$ & $0.002^{\mathrm{b}}$ \\
Acute operation & $6.6(26)$ & $7.2(17)$ & $5.6(9)$ & $0.527^{\mathrm{b}}$ \\
Unspecified & $5.6(22)$ & $6.8(16)$ & $3.8(6)$ & $0.193^{\mathrm{b}}$ \\
\hline
\end{tabular}

IQR interquartile range, SD standard deviation, $C$ confidence interval, ICU intensive care unit, SAPS // simplified acute physiology score II, SOFA sequential organ failure assessment, $\mathrm{CCl}$ Charlson comorbidity index

${ }^{a}$ Independent $t$ test

${ }^{b}$ Pearson's chi-square

'Mann-Whitney U test

${ }^{\mathrm{d}}$ Fisher's exact test

$p=0.031$ ), and severe circulatory failure more frequently (52.3 vs. $39.0 \%, p=0.010$ ) than females.

\section{Length of stay}

The overall median ICU-LOS and hospital-LOS were 1.8 and 11.3 days, respectively. The median LOS for ICU non-survivors was 1.3 days (see Table 1 ). Among all patients, $26.8 \%$ stayed less than 1 day in the ICU.

\section{Ventilator support}

Of 395 patients, $61.3 \%$ received mechanical ventilator support for a median time of 1.2 days. Of the hospital non-survivors, $75.0 \%(n=120)$ received mechanical ventilator support (Table 1). A fraction of $69.2 \%(n=$ 83) of these 120 patients had life-sustaining treatment limitation decisions.

\section{Severity scores and severe organ dysfunction}

Overall, the mean SAPS II and mean maximal SOFA scores were 44.3 and 7.8, respectively. Hospital nonsurvivors had a mean SAPS II of 49.5 and a mean maximal SOFA score of 9.5 (Table 1). All patients with maximal SOFA scores $\geq 17$ died in the ICU; all those with scores $\geq 16$ died during the hospital stay.

\section{Comorbidity}

Overall mean Charlson comorbidity index was 2.6. Patients admitted for planned surgery showed the highest index score among the admission categories (3.2). Only $12.3 \%$ of the patients had no preexisting comorbidity (see Table 1 and 2).

\section{Diagnostic groups}

The most frequent cause for ICU admission was respiratory failure $(28 \%)$. Respiratory failure was most common in the planned surgery group (44\%; Table 2 ).

\section{Survival and predictors of mortality Short-term survival}

The overall ICU and hospital survival were 75.9 and $59.5 \%$, respectively. Of the ICU non-survivors, $63.2 \%$ died within 2 days after ICU admission $(n=60)$, and $68.3 \%$ of these patients had life-sustaining treatment (LST) limitations $((n=41)$; withholding $60.0 \%$ and withdrawal $51.7 \%$ ). The SMR was 1.06, with large differences between the planned surgery (0.55) and unplanned surgery (1.15) groups. For survival at 30, 90, and 180 days, see Table 2.

Predictors of high ICU mortality were age, mechanical ventilator support, SAPS II, maximum SOFA, and multitrauma with head injury. Increased hospital mortality was predicted by an unplanned surgical admission (Table 3).

\section{Long-term survival (1 year and longer)}

The overall 1- and 2-year survival rates were 42.0 and $36.6 \%$, respectively. After 5 years, $22.2 \%$ of all patients remained alive. A comparison between patients $(n=$ $395)$ and the general population greater than 80 years old in Norway $(n=426773)$ showed excess mortality among patients in the first year, with an adjusted mortality rate of 6.35 (95 \% CI 5.58-7.23). After the first year, the survival rates were similar between groups; patients had an adjusted mortality rate during the second year of 1.34 (95 \% CI 0.86-2.07; Fig. 2). Among patients alive after 1 year, the mean survival time, starting from the 1year point, was 5.1 years.

Respiratory failure and isolated head injury were independent predictors of 1-year mortality (Table 3 ).

\section{Survival at follow-up}

At follow-up (January 2014), 322 (81.5\%) patients had died, including 160 during the hospital stay and 162 after hospital discharge. Seventy-three patients (18.5 \%) survived, with a mean age of 86.9 years at follow-up. The median time from hospital discharge to follow-up was 3.3 years (range 1-13.8 years; Fig. 1). The survivors at follow-up $(n=73)$ had, compared to hospital survivors not alive at follow-up $(n=162)$, similar ICU-LOS (1.9 vs. 1.8 days; $p=0.465$ ), fraction of ventilator support ( 52.1 vs. $51.9 \% ; p=0.977$ ), severity of illness (SAPS II 43.2 vs. $39.4, p=0.658$; max. SOFA 6.5 vs. $6.7, p=$ 0.313 ), and comorbidity (Charlson comorbidity index 2.4 vs. 2.8, $p=0.156$; Additional file 1: Table S1). However, hospital survivors not alive at follow-up had a lower median survival after hospital discharge (3.1 years), compared to the follow-up of 3.4 years in survivors. 
Table $\mathbf{2}$ Characteristics of SAPS II admission categories

\begin{tabular}{|c|c|c|c|c|c|}
\hline & $\begin{array}{l}\text { Planned surgery } \\
(n=50)\end{array}$ & $\begin{array}{l}\text { Unplanned surgery } \\
(n=213)\end{array}$ & $\begin{array}{l}\text { Medical reasons } \\
(n=132)\end{array}$ & Total $(n=395)$ & $p$ value \\
\hline Age, mean $\pm S D$ & $83.5 \pm 2.7$ & $84.0 \pm 2.7$ & $83.5 \pm 3.2$ & $83.8 \pm 2.9$ & $0.217^{\mathrm{a}}$ \\
\hline Male, \% & 64.0 & 60.6 & 60.6 & 61.0 & $0.889^{b}$ \\
\hline \multicolumn{6}{|l|}{ Length of stay (LOS), median (IQR) } \\
\hline ICU-LOS & $2.0(1.0-4.4)$ & $2.2(1.0-5.0)$ & $1.2(0.6-2.6)$ & $1.8(0.9-3.9)$ & $<0.001^{\mathrm{b}}$ \\
\hline Hospital LOS & $15.1(10.2-26.2)$ & $12.9(4.3-20.6)$ & $6.5(2.0-14.3)$ & $11.3(4.0-19.3)$ & $<0.001^{b}$ \\
\hline \multicolumn{6}{|l|}{ Ventilator support } \\
\hline Mechanical ventilator support, \% (n) & $48.0(24)$ & $69.5(148)$ & $53.0(70)$ & $61.3(242)$ & $0.001^{b}$ \\
\hline $\begin{array}{l}\text { Mechanical ventilator support time, } \\
\text { median (IQR) }\end{array}$ & $1.1(0.4-3.7)$ & $1.3(0.5-3.8)$ & $0.9(0.3-2.0)$ & $1.2(0.5-3.3)$ & $0.050^{b}$ \\
\hline Non-invasive ventilator support, \% (n) & $44.0(22)$ & $33.8(72)$ & $28.0(37)$ & $29.6(47)$ & $0.119^{b}$ \\
\hline Non-invasive ventilator support time, median (IQR) & $1.2(0.5-2.6)$ & $2.0(0.6-3.0)$ & $1.0(0.3-2.0)$ & $1.3(0.4-2.5)$ & $0.056^{\mathrm{b}}$ \\
\hline \multicolumn{6}{|l|}{ Severity score, mean $\pm S D$} \\
\hline SAPS $\|$ & $\begin{array}{l}39.0 \pm 13.2 \\
(n=47)\end{array}$ & $\begin{array}{l}44.6 \pm 14.8 \\
(n=212)\end{array}$ & $\begin{array}{l}45.6 \pm 15.6 \\
(n=131)\end{array}$ & $\begin{array}{l}44.3 \pm 15.0 \\
(n=390)\end{array}$ & $0.030^{\mathrm{a}}$ \\
\hline Max. SOFA & $6.3 \pm 4.1(n=47)$ & $8.3 \pm 3.6(n=212)$ & $7.5 \pm 3.8(n=130)$ & $7.8 \pm 3.8(n=389)$ & $0.002^{\mathrm{a}}$ \\
\hline \multicolumn{6}{|l|}{ Comorbidity } \\
\hline Charlson comorbidity index, mean \pm SD & $3.2 \pm 1.8(n=50)$ & $2.5 \pm 2.0(n=209)$ & $2.5 \pm 1.7(n=131)$ & $2.6 \pm 1.9(n=390)$ & $0.050^{\mathrm{a}}$ \\
\hline \multicolumn{6}{|l|}{ Charlson comorbidity index (CCl) categories, \% (n) } \\
\hline None $(\mathrm{CCl} 0)$ & $4.0(2)$ & $13.4(28)$ & $13.7(18)$ & $12.3(48)$ & $0.159^{b}$ \\
\hline Mild (CCI 1-2) & $34.0(17)$ & $43.1(90)$ & $39.7(52)$ & $40.8(159)$ & $0.436^{\mathrm{b}}$ \\
\hline Moderate (CCl 3-4) & $46.0(23)$ & $26.3(55)$ & $36.6(48)$ & $32.3(126)$ & $0.009^{b}$ \\
\hline Severe $(\mathrm{CCl} \geq 5)$ & $16.0(8)$ & $17.2(36)$ & $9.9(13)$ & $14.6(57)$ & $0.163^{b}$ \\
\hline \multicolumn{6}{|l|}{ Severe organ dysfunction, \% (n) } \\
\hline Respiration & $68.0(34)$ & $71.8(153)$ & $56.8(75)$ & $66.3(262)$ & $0.016^{\mathrm{b}}$ \\
\hline Circulation & $38.0(19)$ & $53.1(113)$ & $40.9(54)$ & $47.1(186)$ & $0.035^{b}$ \\
\hline Renal & $30.0(15)$ & $28.6(61)$ & $26.5(35)$ & $28.1(111)$ & $0.868^{\mathrm{b}}$ \\
\hline CNS & $20.0(10)$ & $23.5(50)$ & $32.6(43)$ & $26.1(103)$ & $0.100^{b}$ \\
\hline Coagulation & $16.0(8)$ & $8.5(18)$ & $9.8(13)$ & $9.9(39)$ & $0.273^{b}$ \\
\hline Liver & $2.0(1)$ & $0.0(0)$ & $3.0(4)$ & $1.3(5)$ & $0.034^{d}$ \\
\hline \multicolumn{6}{|l|}{ Survival, \% (n) } \\
\hline $\mathrm{ICU}$ & $90.0(45)$ & $74.2(158)$ & $73.5(97)$ & $75.9(300)$ & $0.045^{b}$ \\
\hline Hospital & $84.0(42)$ & $54.9(117)$ & $57.6(76)$ & $59.5(235)$ & $0.001^{b}$ \\
\hline 30 days & $86.0(43)$ & $51.2(109)$ & $54.5(72)$ & $56.7(224)$ & $<0.001^{\mathrm{b}}$ \\
\hline 90 days & $82.0(41)$ & $44.6(95)$ & $50.8(67)$ & $51.4(203)$ & $<0.001^{b}$ \\
\hline 180 days & $74.0(37)$ & $40.4(86)$ & $47.7(63)$ & $47.1(186)$ & $<0.001^{\mathrm{b}}$ \\
\hline 1 year & $68.0(34)$ & 37.1 (79) & $40.2(53)$ & $42.0(166)$ & $<0.001^{\mathrm{b}}$ \\
\hline 2 years & $59.9(28)$ & $33.1(64)$ & $33.6(39)$ & $36.6(130)$ & $0.001^{\mathrm{b}}$ \\
\hline 3 years & $48.4(15)$ & $27.8(50)$ & $29.9(32)$ & $31.2(96)$ & $0.088^{b}$ \\
\hline 5 years & $32.8(7)$ & $18.6(28)$ & $23.7(22)$ & $22.2(55)$ & $0.290^{b}$ \\
\hline \multicolumn{6}{|l|}{ Diagnostic groups, \% ( $n)$} \\
\hline Respiratory failure & $44.0(22)$ & $24.4(52)$ & $28.0(37)$ & $28.1(111)$ & $0.021^{\mathrm{b}}$ \\
\hline Circulatory failure & $8.0(4)$ & $8.5(18)$ & $7.6(10)$ & $8.1(32)$ & $0.959^{b}$ \\
\hline Combined respiratory and circulatory failure & $8.0(4)$ & $12.7(27)$ & $7.6(10)$ & $10.4(41)$ & $0.270^{\mathrm{b}}$ \\
\hline
\end{tabular}


Table 2 Characteristics of SAPS II admission categories (Continued)

\begin{tabular}{|c|c|c|c|c|c|}
\hline Neurologic failure & $2.0(1)$ & $7.0(15)$ & $18.2(24)$ & $10.1(40)$ & $<0.001^{b}$ \\
\hline Isolated head injury & $0.0(0)$ & $1.9(4)$ & $4.5(6)$ & $2.5(10)$ & $0.165^{d}$ \\
\hline Sepsis & $4.0(2)$ & $8.9(19)$ & $10.6(14)$ & $8.9(35)$ & $0.308^{d}$ \\
\hline Gastroenterological failure & $0(0)$ & $5.6(12)$ & $5.3(7)$ & $4.8(19)$ & $0.276^{d}$ \\
\hline Multiple organ failure & $4.0(2)$ & $7.5(16)$ & $3.0(4)$ & $5.6(22)$ & $0.205^{d}$ \\
\hline Multitrauma without head injury & $0.0(0)$ & $5.6(12)$ & $2.3(3)$ & $3.8(15)$ & $0.107^{d}$ \\
\hline Multitrauma with head injury & $0.0(0)$ & $2.3(5)$ & $3.0(4)$ & $2.3(9)$ & $0.638^{d}$ \\
\hline Planned surgery & $18.0(9)$ & $1.4(3)$ & $0.8(1)$ & $3.3(13)$ & $<0.001^{d}$ \\
\hline Acute operation & $2.0(1)$ & $10.3(22)$ & $2.3(3)$ & $6.6(26)$ & $0.004^{d}$ \\
\hline Unspecified & $10.0(5)$ & $3.8(8)$ & $6.8(9)$ & $5.6(22)$ & $0.150^{d}$ \\
\hline SMR (95 \% Cl) & $\begin{array}{l}0.55(0.28-1.11) \\
(n=47)\end{array}$ & $\begin{array}{l}1.15(0.94-1.40) \\
(n=212)\end{array}$ & $\begin{array}{l}1.05(0.81-1.37) \\
(n=131)\end{array}$ & $\begin{array}{l}1.06(0.90-1.23) \\
(n=390)\end{array}$ & \\
\hline
\end{tabular}

Survival times were derived from the life table method

IQR interquartile range, SD standard deviation, $C I$ confidence interval, ICU intensive care unit, SAPS /I simplified acute physiology score II, SOFA sequential organ failure assessment, $\mathrm{CCl}$ Charlson comorbidity index, SMR standardized mortality ratio

With ANOVA (analysis of variance)

${ }^{b}$ Pearson's chi-square test

'With Kruskal-Wallis test

${ }^{\mathrm{d}}$ Fisher's exact test

\section{SAPS II admission categories}

Patients admitted for planned surgery had significantly higher survival rates than those admitted for medical reasons and unplanned surgery up to 3 years after ICU admittance (Table 2). The median survival times were 33.4 months (95 \% CI 21.2-45.6) for planned surgery, 1.2 months (95 \% CI 0.0-2.7) for unplanned surgery, and 2.7 months (95 \% CI 0.0-9.1) for medical admissions (Fig. 3).

\section{Health-related quality of life}

The EQ-5D questionnaire was sent to the 73 patients who were alive at follow-up. The response rate was $83.6 \%$ ( $n=$ 61 ), but one questionnaire was incomplete, and two questionnaires had no patient identity. Fourteen patients responded to the questionnaire by telephone. The response rate in the control group was $47.7 \%$ (179/375), constituting 2.5 controls per survivor at follow-up. HRQOL was similar between patients and the general population and among the admission categories (Table 4).

\section{Life-sustaining treatment limitation in hospital non- survivors}

Of the ICU non-survivors, $70.5 \%(n=67)$ had treatmentlimitation decisions; withholding $68.4 \%(n=65)$ and withdrawal $51.6 \%(n=49)$. The majority of these LST limitation decisions were taken within the first 2 days after ICU admission $(61.2 \%(n=41))$. Post ICU $72.3 \%(n=47)$ of the hospital non-survivors had treatment-limitation decisions; withholding $72.3 \%(n=47)$ and withdrawal $32.3 \%$ $(n=21)$. We lack information on LST decisions in six ICU non-survivors and two ICU survivors.

\section{Discussion}

This study establishes three major results. First, patients who survived the first year after ICU admittance showed long-term survival rates similar to those of the normal Norwegian octogenarian population. The HRQOL of long-time survivors was comparable to that of an age- and sex-matched general population group. Second, the planned surgery group exhibited higher survival rates than the medical and unplanned surgery groups up to 3 years after ICU admittance. However, at follow-up, HRQOL did not differ among these three groups. Third, high ICU mortality was predicted by age, mechanical ventilator support, SAPS II, maximum SOFA score, and multitrauma with head injury. High hospital mortality was predicted by an unplanned surgical admission. Respiratory failure and isolated head injury were independent predictors of 1-year mortality. The majority of the ICU non-survivors died within 2 days, where most of these had life-sustaining treatment (LST) limitations. Almost three quarters of the hospital non-survivors had treatment-limitation decisions.

Our finding of age as an independent predictor of ICU mortality contrasts with several previous studies $[14,15]$. Conflicting results about the impact of age on outcome for older patients in the ICU may be explained by variations in adjustments for severity and comorbidities among different studies. Moreover, in some institutions, older individuals may have been denied ICU admission, based on advanced age [16]. In addition, treatment is often withheld for older ICU patients with severe comorbidity [17]. In our study, advanced age may also have influenced preferences in decision-making among patients, relatives, or caregivers. The influence of age on mortality must be adjusted for severity of illness. 
Table 3 Predictors of mortality in the ICU, in hospital, and at 1 year after admission

\begin{tabular}{|c|c|c|c|}
\hline & $\begin{array}{l}\text { ICU mortality } \\
(n=389)\end{array}$ & $\begin{array}{l}\text { Hospital mortality for } \\
\text { ICU survivors }(n=294)\end{array}$ & $\begin{array}{l}1 \text {-year mortality for hospital } \\
\text { survivors }(n=230)\end{array}$ \\
\hline & Adjusted HR (95 \% Cl) & Adjusted HR (95 \% CI) & Adjusted HR (95\% Cl) \\
\hline Age, years & $1.10(1.03-1.18)^{a}$ & & \\
\hline \multicolumn{4}{|l|}{ Male } \\
\hline \multicolumn{4}{|l|}{ Ventilator support } \\
\hline Mechanical ventilator support & $1.99(1.10-3.60)^{\mathrm{a}}$ & $1.40(0.81-2.43)$ & \\
\hline Non-invasive ventilator support & $0.87(0.51-1.49)$ & & \\
\hline \multicolumn{4}{|l|}{ Severity score, mean } \\
\hline SAPS $\|$ & $1.03(1.01-1.04)^{\mathrm{a}}$ & $1.01(0.99-1.03)$ & $1.01(0.99-1.03)$ \\
\hline Max. SOFA & $1.20(1.10-1.31)^{\mathrm{a}}$ & $1.03(0.95-1.12)$ & \\
\hline \multicolumn{4}{|l|}{ Comorbidity } \\
\hline None $(\mathrm{CCl} 0)$ & 1.00 & & 1.00 \\
\hline Mild (CCI 1-2) & $0.68(0.35-1.30)$ & & $1.02(0.43-2.46)$ \\
\hline Moderate (CCl 3-4) & $0.53(0.25-1.11)$ & & $1.06(0.42-2.65)$ \\
\hline Severe $(\mathrm{CCl} \geq 5)$ & $0.53(0.23-1.25)$ & & $2.09(0.99-5.39)$ \\
\hline \multicolumn{4}{|l|}{ Severe organ dysfunction } \\
\hline Respiration & $1.05(0.55-1.97)$ & & \\
\hline Circulation & $0.76(0.39-1.48)$ & & \\
\hline Renal & $1.50(0.88-2.54)$ & & \\
\hline CNS & $1.19(0.71-1.99)$ & & \\
\hline \multicolumn{4}{|l|}{ Coagulation } \\
\hline \multicolumn{4}{|l|}{ Liver } \\
\hline \multicolumn{4}{|l|}{ Admission categories } \\
\hline Planned surgery & 1.00 & 1.00 & 1.00 \\
\hline Unplanned surgery & $1.40(0.54-3.65)$ & $3.46(1.06-11.24)^{\mathrm{a}}$ & $2.02(0.88-4.64)$ \\
\hline Medical reasons & $2.11(0.80-5.58)$ & $3.17(0.94-10.76)$ & $1.97(0.83-4.70)$ \\
\hline \multicolumn{4}{|l|}{ Diagnostic groups } \\
\hline Respiratory failure & $1.03(0.55-1.90)$ & & $1.86(1.13-3.07)^{\mathrm{a}}$ \\
\hline \multicolumn{4}{|l|}{ Circulatory failure } \\
\hline \multicolumn{4}{|c|}{ Combined respiratory and circulatory failure } \\
\hline Neurologic failure & & $1.67(0.86-3.25)$ & \\
\hline Isolated head injury & $1.56(0.58-4.18)$ & & $9.12(2.44-34.14)^{\mathrm{a}}$ \\
\hline Sepsis & $1.20(0.63-2.69)$ & & \\
\hline \multicolumn{4}{|l|}{ Gastroenterological failure } \\
\hline Multiple organ failure & $1.27(0.60-2.69)$ & $1.67(0.64-4.31)$ & \\
\hline \multicolumn{4}{|l|}{ Multitrauma without head injury } \\
\hline Multitrauma with head injury & $2.99(1.04-8.60)^{\mathrm{a}}$ & & \\
\hline \multicolumn{4}{|l|}{ Planned surgery } \\
\hline \multicolumn{4}{|l|}{ Acute operation } \\
\hline Unspecified & & & \\
\hline
\end{tabular}

ICU intensive care unit, SAPS II simplified acute physiology score II, SOFA sequential organ failure assessment, $\mathrm{HR}$ hazard ratio, $\mathrm{Cl}$ confidence interval, CCI Charlson comorbidity index

${ }^{\mathrm{a}}$ Significant differences 


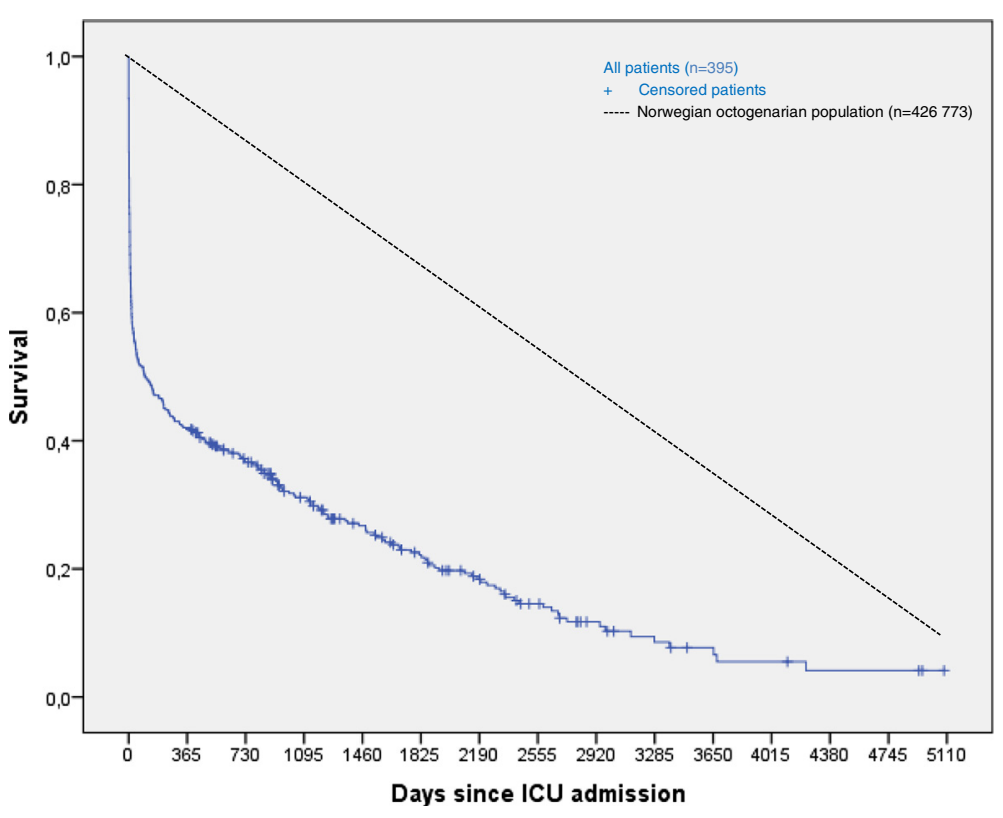

Fig. 2 Kaplan-Meier survival curve for all patients (solid blue line) compared to the Norwegian octogenarian population (dashed black line) in 2000-2013

In general, ICU length of stay is short in Scandinavian countries $[6,7,18]$. One explanation can be the low availability of ICU beds compared to many other European countries $[3,19]$. Also, octogenarians are in general found to have shorter ICU stays than younger patients $[6,20,21]$. This is probably reflected in our study. The overall median ICU-LOS was 1.8 days, which was 3.2-4.2 days shorter than that reported in recent French studies [8, 9]. Also, our ICU and hospital mortality rates were lower than reported in those studies. These findings might be explained by differences in "case-mix" within the SAPS II admission categories, where the French studies included mostly medical cases. However, our medical group had significantly shorter ICU stays (median 1.2 days) than the unplanned surgery group. This finding could not be explained by differences in mortalities or SAPS II scores. Even though ICU-LOS is short in our study, the mean SAPS II scores and mechanical ventilator support rates are comparable to other octogenarian cohort studies $[8,9,14,21-23]$. In general, our survivors had longer ICU stays than non-survivors, due to

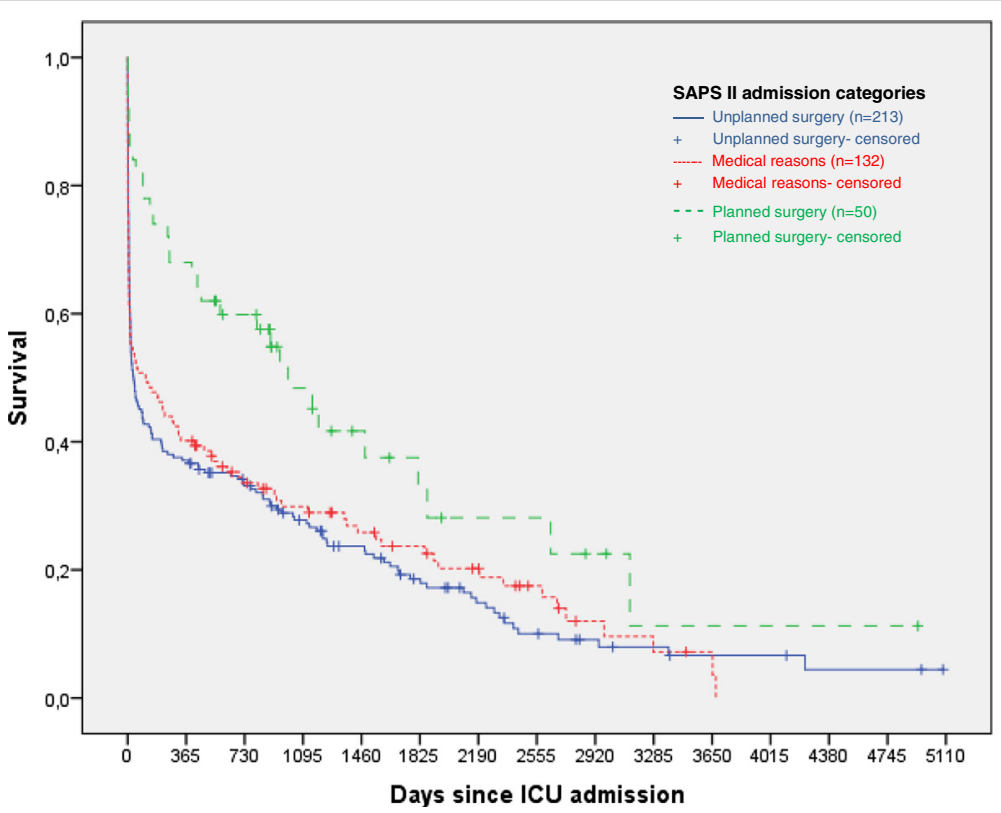

Fig. 3 Kaplan-Meier survival curves of SAPS II admission categories 
Table 4 Comparison of frequency distributions (profiles) of the EQ-5D-3L for patient and control groups

\begin{tabular}{|c|c|c|c|}
\hline Variable & Total patients $(n=58)$ & Control group $(n=179)$ & $p$ value \\
\hline Age, years, mean $\pm S D$ & $87.4 \pm 4.0$ & $86.7 \pm 4.4$ & $0.265^{\mathrm{a}}$ \\
\hline Male, \% (n) & $69.0(40)$ & $66.5(119)$ & $0.726^{\mathrm{b}}$ \\
\hline Mobility, \% (n) & & & $0.504^{c}$ \\
\hline No problem & $41.4(24)$ & $43.6(78)$ & \\
\hline Some problems & $51.7(30)$ & $54.7(98)$ & \\
\hline Confined to bed & $6.9(4)$ & $1.7(3)$ & \\
\hline Self-care, \% (n) & & & $0.957^{c}$ \\
\hline No problem & $75.9(44)$ & 74.9 (134) & \\
\hline Some problems & $15.5(9)$ & $21.8(39)$ & \\
\hline Unable to & $8.6(5)$ & $3.4(6)$ & \\
\hline Usual activities, \% (n) & & & $0.237^{c}$ \\
\hline No problem & $43.1(25)$ & $49.7(89)$ & \\
\hline Some problems & $41.4(24)$ & $41.3(74)$ & \\
\hline Unable to & $15.5(9)$ & $8.9(16)$ & \\
\hline Pain and discomfort, \% (n) & & & $0.229^{c}$ \\
\hline None & $43.1(25)$ & $34.6(62)$ & \\
\hline Moderate & $51.7(30)$ & $58.1(104)$ & \\
\hline Extreme & $5.2(3)$ & $7.3(13)$ & \\
\hline Anxiety and depression, \% (n) & & & $0.258^{\mathrm{C}}$ \\
\hline None & $77.6(45)$ & $69.8(125)$ & \\
\hline Moderate & $20.7(12)$ & $27.9(50)$ & \\
\hline Extreme & $1.7(1)$ & $2.2(4)$ & \\
\hline EQ index, mean $\pm S D$ & $0.71 \pm 0.28$ & $0.73 \pm 0.23$ & $0.924^{c}$ \\
\hline EQ VAS, mean \pm SD & $63.9 \pm 20.3(n=53)$ & $67.7 \pm 22.0(n=170)$ & $0.219^{c}$ \\
\hline
\end{tabular}

$S D$ standard deviation, EQ EuroQol, VAS visual analog scale (range 0-100)

andependent $t$ test

bPearson's chi-square

${ }^{c}$ Mann-Whitney U test

death shortly after ICU admittance (63.2 \% within 2 days). The large proportion of LST limitations among ICU nonsurvivors during the first 2 days after ICU admission may contribute to the short length of stay. Our data could indicate that ICU physicians limit the intensity of lifesustaining treatment if there is no improvement in the condition of the octogenarian patient within the first 2 days after ICU admission. Although we lack data on triage decisions prior to ICU admission, we might speculate that a more thorough pre-ICU triage process could have decreased the rather high fraction of LST limitation decisions by rejecting patients who probably would not benefit from ICU treatment.

After the first year, we found our ICU patients to have survival rates similar to those of the general octogenarian population. Interestingly, Roch et al. found a similar trend after 2 years [9]. The low 1-year survival rate may indicate that many aged patients did not benefit from ICU treatment. Therefore, we need better predictors to determine which patients are likely to gain long-term benefit from
ICU treatment. Several studies have reported prognostic factors for short- and long-term mortality among older individuals $[14,17,24]$. In general, short-term mortality is most frequently predicted by severity scores and the number of organ failures [17]. Commonly used prognostic models for aged patients in the ICU lack calibration. Nevertheless, our study showed that severity scores were good predictors for ICU mortality, in addition to age, mechanical ventilator support, and multitrauma with head injury. One study developed a prognostic model for predicting in-hospital mortality in older patients in the ICU, and found low Glacow coma scale (GCS) scores to be strongly related to short-term mortality [24]. Several other studies have reported that brain injury is associated with poor outcomes in older patients [25,26]. Comorbidity is also found to be a predictor of long-term survival in some octogenarian ICU studies [9, 14]. However, these studies used the McCabe classification, where comorbidity is based on the presence of underlying fatal diseases. In our study, we found no association between long-term mortality and 
comorbidity, using the Charlson comorbidity index. This is supported in other studies [5, 27], also using Charlson comorbidity index. The regression analysis of ICU mortality showed decreasing hazard of death with increasing comorbidity. This was probably mainly due to admission of patients with no comorbidity who suffered severe trauma and bleeding events, with high mortality. We might speculate that the admission policy of these patients was more liberal due to lack of comorbidity, even if the prospects of survival was low, compared to patients with higher comorbidity. In our opinion, comorbidity is not a very useful predictor for ICU mortality in general nor for the elderly population.

To our knowledge, this study is the first to report HRQOL in older patients over a 13-year post-ICU followup. We found similar HRQOLs in ICU survivors and the general Norwegian octogenarian population at follow-up. Other recent studies on HRQOL in older ICU survivors have reported impaired physical function $[9,28]$. De Rooij et al. found that patients had more problems with usual activities and lower mean EQ VAS scores than the general British population [29]. In contrast, Tabah et al. reported a similar HRQOL, or better in some domains, compared to a matched general population [8]. Good HRQOL perceptions, despite physical impairment, could be due to lower expectations of life after critical illness. However, HRQOL evaluations may be prone to selection bias, because responders may represent healthier patients. Our study revealed that non-responders and responders had similar severity scores and similar fractions of severe organ failures. But non-responders were evaluated at slightly longer times after hospital discharge (median 4.6 vs. 3.3 years; $p=0.350$ ). Patients alive after 1 year had a mean further survival time of 5.1 years. Furthermore, survivors at follow-up had longer time to follow-up compared to the median survival in hospital survivors not alive at followup. Nevertheless, these groups were otherwise comparable, and we can speculate that hospital survivors no longer alive had about the same HRQOL as survivors at follow-up, at least for much of the time left (Additional file 1: Table S1).

Very few studies have reported outcomes for aged patients in different SAPS II admission categories. De Rooij et al. reported higher short- and long-term survival in patients admitted for planned surgery compared to those admitted for medical reasons and unplanned surgery, with a mean follow-up of 3.6 years [22]. Our results supported that finding, but only up to 3 years after ICU admittance. Thereafter, long-term survival was similar among the groups. We also found that an unplanned surgery admission could predict high hospital mortality in ICU survivors.

\section{Limitations}

This study has several limitations. First, it was partly a retrospective study and clinical data were confined to those registered in the ICU database. Thus, we had no information about triage decisions made before ICU admission. Variability in these decisions may influence the results [3032]. Second, the long inclusion period could contain changes in admission policy and medical practice. However, the catchment area and basic functions of the hospital remained the same during the study period, with a slowly growing population and all medical services except organ transplant surgery offered. There were no large changes in practice or organizational changes in the ICU during the study period. Third, due to our single-center study design, the group sizes were relatively small. In particular, the number of patients for HRQOL assessment was limited ( $n=$ 73 ); this is common in single-center studies of aged ICU populations. Furthermore, the HRQOLs of different groups were evaluated at different follow-up times. Nevertheless, every patient was followed-up after at least 1 year, the recommended minimum [33]. Furthermore, the high response rate for the EQ-5D questionnaire $(n=58,79.5 \%)$ provided valuable $\mathrm{HRQOL}$ information among older, long-term ICU survivors in Norway, particularly compared to the age- and gender-matched control group. We evaluated HRQOL once in each patient; thus, we did not study changes in HRQOL over time. Ideally, a baseline measurement should be made before the ICU stay. Finally, we had no information on living status or cognitive functions.

\section{Conclusions}

One-year survival was $42.0 \%$, with further survival comparable to the general octogenarian population. HRQOL in survivors was comparable with an age- and sexmatched general population, with a follow-up of 1-13.8 years. Up to 3 years after ICU admittance, patients admitted for planned surgery had better short- and long-term outcomes than those admitted for medical reasons and unplanned surgery. The majority of the ICU nonsurvivors died within 2 days, and most of these had life-sustaining treatment (LST) limitations. Almost three quarters of the hospital non-survivors had treatmentlimitation decisions. Our results indicate that older ICU patients have poor short-term outcomes due to high mortalities, but good long-term outcomes in those who survive beyond 1 year. Predictors identified in this study may facilitate triage decisions in older patients regarding ICU treatment. Future research should focus on improving prognostic models for aged patients.

\section{Key messages}

- One-year survival was $42.0 \%$; thereafter, survival was comparable to that of the general octogenarian population.

- HRQOL in our survivors at follow-up $(n=58)$ was comparable with an age- and gender-matched 
general population $(n=179)$, for a follow-up of $1-13.8$ years.

- Patients admitted for planned surgery had better short- and long-term survival rates than those admitted for medical reasons or unplanned surgeries for three years after ICU admittance.

- The majority of the ICU non-survivors died within 2 days (63.2\%), and most of these had life-sustaining treatment (LST) limitations (68.3\%).

\section{Additional file}

Additional file 1: Table S1. Differences in characteristics between hospital survivors not alive at follow-up $(n=162)$ and survivors at follow-up $(n=73)$.

\section{Abbreviations}

HRQOL: health-related quality of life; ICU: intensive care unit; LOS: length of stay; SAPS II: simplified acute physiology score II; SOFA: sequential organ failure assessment score; CCl: Charlson comorbidity index; SMR: standardized mortality ratio; SD: standard deviation; Cl: confidence interval; IQR: interquartile range; ANOVA: analysis of variance; HR: hazard ratio; LST: life-sustaining.

\section{Competing interests}

The authors declare that they have no competing interests.

\section{Authors' contributions}

FHA conceived the study, participated in its coordination, performed the statistical analyses, and drafted the manuscript. RK, HF, and PK made substantial contribution to the conception and design of the study and helped to draft the manuscript. UR participated in the design of the study and supervised during the statistical analyses. All authors read and approved the final manuscript.

\section{Acknowledgements}

The study was supported by a grant from the Møre and Romsdal Health Trust.

\section{Author details}

'Department of Anesthesia and Intensive Care, Møre and Romsdal Health Trust, Ålesund Hospital, 6026 Ålesund, Norway. ${ }^{2}$ Department of Circulation and Medical Imaging, Faculty of Medicine, Norwegian University of Science and Technology, Pb. 8905, 7491 Trondheim, Norway. ${ }^{3}$ Department of Anesthesia and Intensive Care, Haukeland University Hospital, Jonas Lies vei 65, 5021 Bergen, Norway. ${ }^{4}$ University of Bergen, Pb. 7800, 5200 Bergen, Norway. ${ }^{5}$ Department of Anesthesia and Intensive Care, St. Olavs Hospital, Prinsesse Kristinas gate 3, 7030 Trondheim, Norway. ${ }^{6}$ Nord-Trøndelag Health Trust, Pb. 333, 7601 Levanger, Norway. ${ }^{7}$ The Public Health Agency of Sweden, 83140 Østersund, Sweden.

\section{Received: 5 March 2015 Accepted: 19 May 2015}

\section{Published online: 03 June 2015}

\section{References}

1. Nguyen $Y L$, Angus DC, Boumendil A, Guidet B. The challenge of admitting the very elderly to intensive care. Annals of intensive care. 2011;1(1):29.

2. Laake JH, Dybwik K, Flaatten HK, Fonneland IL, Kvale R, Strand K. Impact of the post-World War II generation on intensive care needs in Norway. Acta Anaesthesiol Scand. 2010;54(4):479-84.

3. Flaatten $\mathrm{H}$. The impact of age in intensive care. Acta Anaesthesiol Scand. 2014;58(1):3-4

4. Bagshaw SM, Webb SA, Delaney A, George C, Pilcher D, Hart GK, et al. Very old patients admitted to intensive care in Australia and New Zealand: a multi-centre cohort analysis. Crit Care. 2009:13(2):R45.

5. Nielsson MS, Christiansen CF, Johansen MB, Rasmussen BS, Tonnesen E, Norgaard M. Mortality in elderly ICU patients: a cohort study.

Acta Anaesthesiol Scand. 2014;58(1):19-26.
6. Andersen $\mathrm{FH}$, Kvale R. Do elderly intensive care unit patients receive less intensive care treatment and. Acta Anaesthesiol Scand. 2012;56(10):1298-305.

7. Reinikainen M, Uusaro A, Niskanen M, Ruokonen E. Intensive care of the elderly in Finland. Acta Anaesthesiol Scand. 2007;51(5):522-9.

8. Tabah A, Philippart F, Timsit JF, Willems V, Francais A, Leplege A, et al. Quality of life in patients aged 80 or over after ICU discharge. Crit Care. 2010;14(1):R2.

9. Roch A, Wiramus S, Pauly V, Forel JM, Guervilly C, Gainnier M, et al. Long-term outcome in medical patients aged 80 or over following admission to an intensive care unit. Crit Care. 2011:15(1):R36.

10. Le Gall JR, Lemeshow S, Saulnier F. A new Simplified Acute Physiology Score (SAPS II) based on a European/North American multicenter study. JAMA. 1993;270(24):2957-63.

11. Vincent JL, Moreno R, Takala J, Willatts S, De Mendonca A, Bruining H, et al. The SOFA (Sepsis-related Organ Failure Assessment) score to describe organ dysfunction/failure. On behalf of the Working Group on Sepsis-Related Problems of the European Society of Intensive Care Medicine Intensive Care Med. 1996;22(7):707-10.

12. Charlson ME, Pompei $P$, Ales KL, MacKenzie CR. A new method of classifying prognostic comorbidity in longitudinal studies: development and validation. J Chronic Dis. 1987:40(5):373-83.

13. Group EQ. EuroQol-a new facility for the measurement of health-related quality of life. Health Policy. 1990;16(3):199-208.

14. Boumendil A, Maury E, Reinhard I, Luquel L, Offenstadt G, Guidet B. Prognosis of patients aged 80 years and over admitted in medical intensive care unit. Intensive Care Med. 2004;30(4):647-54.

15. Somme D, Maillet JM, Gisselbrecht M, Novara A, Ract C, Fagon JY. Critically ill old and the oldest-old patients in intensive care: short- and long-term outcomes. Intensive Care Med. 2003:29(12):2137-43.

16. Garrouste-Orgeas M, Timsit JF, Montuclard L, Colvez A, Gattolliat O, Philippart F, et al. Decision-making process, outcome, and 1-year quality of life of octogenarians referred for intensive care unit admission. Intensive Care Med. 2006;32(7):1045-51.

17. de Rooij SE, Abu-Hanna A, Levi M, de Jonge E. Factors that predict outcome of intensive care treatment in very elderly patients: a review. Crit Care. 2005:9(4):R307-14

18. Strand K, Walther SM, Reinikainen M, Ala-Kokko T, Nolin T, Martner J, et al. Variations in the length of stay of intensive care unit nonsurvivors in three Scandinavian countries. Crit Care. 2010;14(5):R175.

19. Rhodes A, Ferdinande P, Flaatten H, Guidet B, Metnitz PG, Moreno RP. The variability of critical care bed numbers in Europe. Intensive Care Med. 2012;38(10):1647-53.

20. Kaarlola A, Tallgren M, Pettila V. Long-term survival, quality of life, and quality-adjusted life-years among critically ill elderly patients. Crit Care Med. 2006:34(8):2120-6.

21. Boumendil A, Aegerter P, Guidet B, Network CU-R. Treatment intensity and outcome of patients aged 80 and older in intensive care units: a multicenter matched-cohort study. J Am Geriatr Soc. 2005;53(1):88-93.

22. de Rooij SE, Govers A, Korevaar JC, Abu-Hanna A, Levi M, de Jonge E. Short-term and long-term mortality in very elderly patients admitted to an intensive care unit. Intensive Care Med. 2006:32(7):1039-44.

23. Lerolle N, Trinquart L, Bornstain C, Tadie JM, Imbert A, Diehl JL, et al. Increased intensity of treatment and decreased mortality in elderly patients in an intensive care unit over a decade. Crit Care Med. 2010;38(1):59-64.

24. de Rooij SE, Abu-Hanna A, Levi M, de Jonge E. Identification of high-risk subgroups in very elderly intensive care unit patients. Crit Care. 2007;11(2):R33.

25. Jacobs DG, Plaisier BR, Barie PS, Hammond JS, Holevar MR, Sinclair KE, et al. Practice management guidelines for geriatric trauma: the EAST Practice Management Guidelines Work Group. J Trauma. 2003:54(2):391-416.

26. Thompson HJ, McCormick WC, Kagan SH. Traumatic brain injury in older adults: epidemiology, outcomes, and future implications. J Am Geriatr Soc. 2006:54(10):1590-5.

27. Pavoni V, Gianesello L, Paparella L, Buoninsegni LT, Mori E, Gori G. Outcome and quality of life of elderly critically ill patients: an Italian prospective observational study. Arch Gerontol Geriatr. 2012;54(2):e193-8.

28. Schroder MA, Poulsen JB, Perner A. Acceptable long-term outcome in elderly intensive care unit patients. Dan Med Bull. 2011;58(7):A4297.

29. de Rooij SE, Govers AC, Korevaar JC, Giesbers AW, Levi M, de Jonge E. Cognitive, functional, and quality-of-life outcomes of patients aged 80 and older who survived at least 1 year after planned or unplanned surgery or medical intensive care treatment. J Am Geriatr Soc. 2008;56(5):816-22. 
30. Boumendil A, Angus DC, Guitonneau A-L, Menn A-M, Ginsburg C, Takun K, et al. Variability of intensive care admission decisions for the very elderly. PLoS One. 2012;7(4), e34387.

31. Sprung CL, Artigas A, Kesecioglu J, Pezzi A, Wiis J, Pirracchio R, et al. The Eldicus prospective, observational study of triage decision making in European intensive care units. Part II: intensive care benefit for the elderly. Crit Care Med. 2012;40(1):132-8.

32. Garrouste-Orgeas M, Boumendil A, Pateron D, Aergerter P, Somme D, Simon T, et al. Selection of intensive care unit admission criteria for patients aged 80 years and over and compliance of emergency and intensive care unit physicians with the selected criteria: an observational, multicenter, prospective study. Crit Care Med. 2009;37(11):2919-28.

33. Oeyen SG, Vandijck DM, Benoit DD, Annemans L, Decruyenaere JM. Quality of life after intensive care: a systematic review of the literature. Crit Care Med. 2010;38(12):2386-400

Submit your manuscript to a SpringerOpen ${ }^{\circ}$ journal and benefit from:

- Convenient online submission

- Rigorous peer review

- Immediate publication on acceptance

- Open access: articles freely available online

- High visibility within the field

- Retaining the copyright to your article

Submit your next manuscript at $>$ springeropen.com 